\title{
Selectively Bred Rats Provide a Unique Model of Vulnerability to PTSD-Like Behavior and Respond Differentially to FGF2 Augmentation Early in Life
}

\author{
Katherine E Prater, ${ }^{*, 2}$, Elyse L Aurbach ${ }^{1,2}$, Hanna K Larcinese', Taylor N Smith', Cortney A Turner', \\ Peter Blandino Jr', Stanley J Watson', Stephen Maren ${ }^{3}$ and Huda Akil' \\ 'Molecular and Behavioral Neuroscience Institute, University of Michigan, Ann Arbor, MI, USA; ${ }^{2}$ Neuroscience Graduate Program, University of \\ Michigan, Ann Arbor, MI, USA; ${ }^{3}$ Department of Psychology, Texas A\&M University, College Station TX, USA
}

Individuals respond differently to traumatic experiences, including their propensity to develop posttraumatic stress disorder (PTSD). Understanding individual differences in PTSD vulnerability will allow the development of improved prevention and treatment options. Here we characterized fear conditioning and extinction in rats selectively bred for differences in their locomotor response to a novel environment. Selectively bred high-responder (bHR) and low-responder (bLR) male rats are known to differ in their emotional reactivity on a range of measures of spontaneous anxiety- and depressive-like behaviors. We demonstrate that bHRs have facilitated extinction learning and retention compared with outbred Sprague Dawley rats, whereas bLRs show reduced extinction learning and retention. This indicates that bLRs are more vulnerable to PTSD-like behavior. Fibroblast growth factor 2 (FGF2) has previously been implicated in the development of these behavioral phenotypes and facilitates extinction learning in outbred animals, therefore we examined the effects of early-life FGF2 on bHR and bLR behavior. FGF2 administered on the day after birth facilitated extinction learning and retention in bHRs, but not in bLRs or control rats, during adulthood. This indicates that, under the current fear conditioning paradigm, early-life FGF2 has protective effects only in resilient animals. This stands in contrast to FGF2's ability to protect vulnerable animals in milder tests of anxiety. These results provide a unique animal model of individual differences in PTSD-like behavior, allowing the study of genetic, developmental, and environmental factors in its expression.

Neuropsychopharmacology (2017) 42, 1706-17|4; doi:10.1038/npp.2017.37; published online 29 March 2017

\section{INTRODUCTION}

In the United States, $>50 \%$ of men and women are likely to experience at least one trauma in their lifetimes, yet incidence rates of posttraumatic stress disorder (PTSD) remain at $\sim 8 \%$ (Kessler et al, 1995). This suggests that individual differences in vulnerability have a critical role in the etiology of PTSD. Although considerable effort has been directed at understanding the genetic and behavioral risk factors (Bomyea et al, 2012; Lommen et al, 2013; Orr et al, 2012), we cannot accurately predict which individuals will develop PTSD. Establishing a reliable prediction method has the potential to advance research on therapeutics for PTSD, and developing better animal models may facilitate this effort (Holly and Miczek, 2015).

To this end, we developed a rodent model of individual differences in which rats were selectively bred based on their

* Correspondence: Dr KE Prater, Molecular and Behavioral Neuroscience Institute, University of Michigan, 2008 MBNI Building, 205 Zina Pitcher Place, Ann Arbor, MI 48I09-5720, USA, Tel: 734764 6999, Fax: 734647 4I30, E-mail: keprater@umich.edu

Received 26 August 2016; revised 9 February 2017; accepted II February 2017; accepted article preview online 16 February 2017 locomotion in a novel environment (for a review, see Flagel et al, 2014). Although a typical population of outbred Sprague Dawley rats displays variability in this trait, selective breeding magnified these differences (Stead et al, 2006). Selectively bred 'high responders' (bHRs) display low spontaneous anxiety- and depression-like behavior and greater aggression in a resident-intruder test, while 'low responders' (bLRs) exhibit high anxiety- and depression-like behavior and less aggression (Kerman et al, 2011; Perez et al, 2009; Stead et al, 2006). These selectively bred lines are a model of individual differences in 'temperament,' or stable tendencies across the lifespan, that in humans can predispose individuals to internalizing $v s$ externalizing psychiatric disorders (Khan et al, 2005). These rats offer a unique opportunity to explore how individual differences in emotional responsiveness influence learning and memory processes involved in the etiology of PTSD, including the conditioning and extinction of fear (Maren et al, 2013).

Fear conditioning and extinction are common models of trauma encoding and exposure therapy (Milad et al, 2014). Previous work demonstrates that PTSD patients have reduced extinction learning and retention (Blechert et al, 2007; Milad et al, 2009; Norrholm et al, 2011; Orr et al, 
2000), and reduced extinction learning may be predictive of future PTSD (Lommen et al, 2013). It is currently unknown whether bLRs might provide a useful model of vulnerability to PTSD-like behavior.

Because we can predict the phenotype of offspring with 99\% accuracy, we can test early interventions that may impact behavior throughout life. Previous studies show that FGF2 administration reduces anxiety and depression-like behavior in our selectively bred rats in adulthood, though a single administration on the day after birth selectively reduces anxiety (Perez et al, 2009; Turner et al, 2011; Turner et al, 2008). Moreover, FGF2 was recently shown to be a potent enhancer of fear and extinction learning (Graham and Richardson, 2011): a single dose of FGF2 administered before extinction enhanced extinction learning and retention (Graham and Richardson, 2009). However, it is unknown whether early-life FGF2 influences extinction learning in adulthood or whether FGF2 differentially affects extinction learning across individuals.

We sought to characterize this animal model and test whether early-life administration of FGF2 differentially impacts fear and extinction learning in individuals with dissimilar temperaments. We hypothesized that bHRs and bLRs would display fear learning similar to outbred animals, but bLRs would show significantly reduced extinction learning and retention, indicating a PTSD-like phenotype. We further hypothesized that administration of FGF2 the day after birth would facilitate extinction learning and retention specifically in bLRs.

\section{MATERIALS AND METHODS}

\section{Animals}

Outbred rats. We used 48 male Sprague-Dawley rats (Charles River Laboratories, Chicago, IL, USA).

Selectively bred rats. We used 142 male bHR and bLR animals from generations 36, 40 and 41 of our colony (Stead et al, 2006) (72 bHRs, 70 bLRs). We used 80 male bred intermediate responders (bIRs), a first-generation cross of bHR-bLR parents.

All animals were pair-housed on a 12:12 h light:dark schedule with access to food and water ad libitum. Animals were handled prior to behavioral testing. The Institutional Animal Care and Use Committee at the University of Michigan approved all procedures.

\section{Behavior}

Locomotor and elevated plus maze (EPM) testing. Locomotor and EPM testing were described previously (Aurbach et al, 2015; Stead et al, 2006); see Supplementary Methods. Animals underwent fear conditioning 1 week after EPM testing.

Fear conditioning and extinction. Fear conditioning consisted of five tone $(2 \mathrm{kHz}, 10 \mathrm{~s}, 80 \mathrm{~dB})$ and shock $(0.6 \mathrm{~mA}, 2 \mathrm{~s})$ pairings. The intertrial interval was $1 \mathrm{~min}$. Rats were PND67-PND73 during testing.

Twenty-four hours after conditioning, animals received extinction in a novel context, consisting of 45 tone-alone
$(2 \mathrm{kHz}, 10 \mathrm{~s}, 80 \mathrm{~dB})$ trials with a $30 \mathrm{~s}$ intertrial interval. NoExtinction control animals entered the extinction context but received no tones. Extinction retention was tested $24 \mathrm{~h}$ after extinction (with the same context and protocol as extinction; all animals received tones). Twenty-four hours after extinction retention, animals were returned to the conditioning context to assess contextual fear (ABBA design). See Supplementary Methods.

Behavioral analysis. Videos were analyzed using a computerized system (CleverSys, Reston, VA, USA). Freezing behavior was quantified during a $3 \mathrm{~min}$ pretrial baseline (Pre) and each $10 \mathrm{~s}$ tone during the conditioning, extinction, and extinction retention sessions. Freezing was quantified during the entire $30 \mathrm{~min}$ context retention test. Escape behavior (ie, climbing, jumping, darting, or biting on parts of the chamber) was hand-scored by two trained observers blind to the condition of the animal. See Supplementary Methods.

\section{Early-Life FGF2 Administration}

Administration protocols were based on Turner et al (2011); see Supplementary Methods.

\section{Corticosterone Assay}

Corticosterone was detected using a radioimmunoassay according to the protocol described by the manufacturer (MP Biomedicals, Santa Ana, CA). See Supplementary Methods.

\section{Data Analysis}

Locomotion and EPM data were analyzed using one-way ANOVA. Corticosterone data were analyzed using one-way ANOVA with repeated measures for collection time. Freezing and escape data were analyzed using two-way (phenotype and extinction group) or three-way (phenotype, treatment, and extinction group) ANOVA with repeated measures across trials. All statistics were calculated using SPSS (IBM). When Mauchly's test of sphericity was found to be significant, the conservative Greenhouse-Geisser correction was applied (Jaccard and Becker, 2009). When a given main effect or interaction met criterion for significance $(p<0.05)$, Bonferroni correction was applied to post hoc comparisons.

\section{RESULTS}

\section{Experiment 1}

Selectively bred animals exhibit different defensive behaviors during conditioning. Prior to testing behavioral responses to conditioned fear, we assessed locomotor behavior to verify the phenotypic effects of selective breeding. As expected, there was a significant main effect of phenotype on locomotor response to novelty with bHRs $>$ bIRs $>$ outbreds $>$ bLRs $(\mathrm{F}(3,88)=311.280, p<0.001$; Figure 1a). Post hoc tests demonstrated significant differences between all phenotypes $(p<0.001)$. Because outbred Sprague Dawley rats and bIR rats did not differ in their 
a

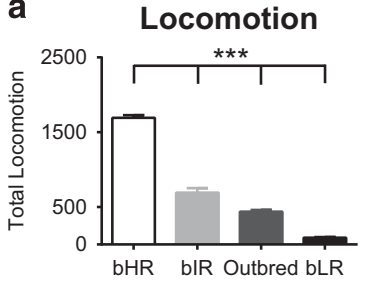

b

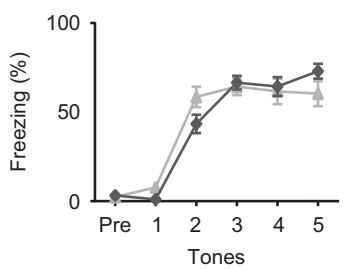

Extinction

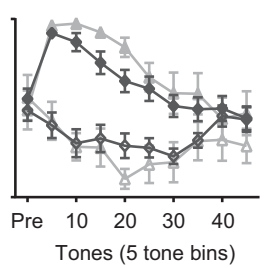

Retention

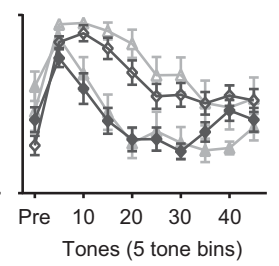

$\vartheta$ Outbred Ext $\triangle$ bIR Ext

- Outbred No-Ext $\quad$ bIR No-Ext

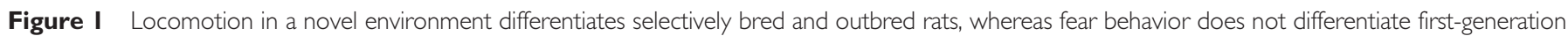

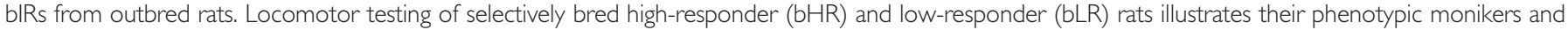

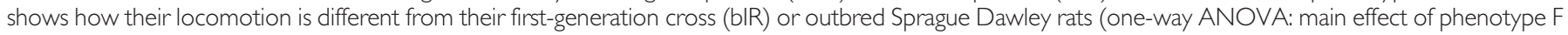

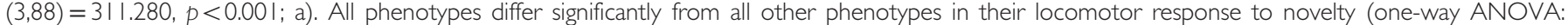

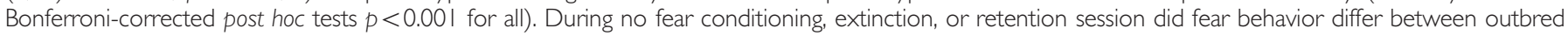

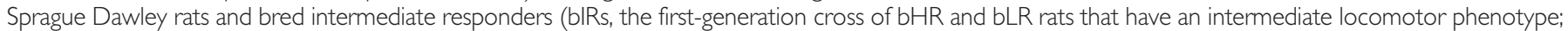

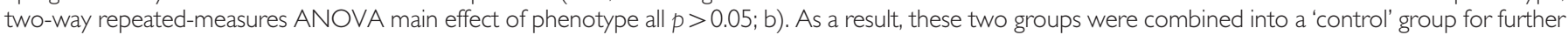

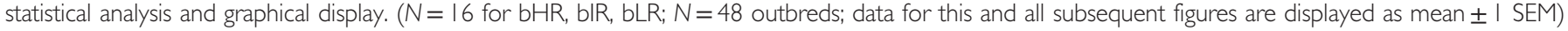
**** $p<0.001$.

behavior during conditioning, extinction, or extinction retention (Figure 1b), they were combined into a 'control' group for all further analyses $(p>0.05$ for all comparisons of outbreds and bIRs).

To characterize fear learning in these different lines of rats, we used a standard fear conditioning paradigm. We observed a main effect of phenotype $(\mathrm{F}(2,90)=23.52, p<0.001$; Figure $2 \mathrm{a}$ and $\mathrm{b})$ : post hoc tests revealed that bHRs froze less than all other phenotypes ( $p<0.001$ for bLR and control rats). There was also a trial by phenotype interaction $(\mathrm{F}(7.08,318.56)$ $=4.78, p<0.001)$ : bHRs had lower freezing during tones $2-5$ (post hoc $p<0.002$, Figure 2a). We confirmed that the fear conditioning paradigm successfully generated learning: the main effect of trial showed that bHR, bLR, and control freezing behavior increased across the session $(\mathrm{F}(3.54,318.56)$ 69.60, $p<0.001$; Supplementary Figure 1A). No other effects reached statistical significance.

To explore why bHR freezing was low during conditioning, all videos were hand-scored by a trained observer blind to phenotype. Analysis of escape behavior during conditioning showed an overall main effect of trial where escape behavior increased across the session $(\mathrm{F}(2.54,106.74)=8.02$, $p<0.001$; Supplementary Figure 1B). Of greater interest was the main effect of phenotype $(\mathrm{F}(2,42)=3.82, p=0.03)$ : bHRs displayed significantly higher escape behavior than bLRs (post hoc $p=0.04$ ) but not controls (post hoc $p=0.12$; Supplementary Figure 1C), suggesting that escape behavior differentiates bHR and bLR responses to fear conditioning. When escape behavior was combined with freezing to generate an overall measure of defensive behavior, the main effect of phenotype remains $(\mathrm{F}(2,42)=7.22, p=0.002$; Figure $2 \mathrm{c}$ and $\mathrm{d}$ ), but the differences between phenotypes during tones 4-5 were eliminated $(p>0.2)$. This indicates that defensive behavior is similar for all phenotypes at the end of the conditioning session, an important marker of similar levels of conditioning prior to extinction learning.

To verify that all animals learned similar levels of fear, we also examined a marker of physiological stress after conditioning. Corticosterone levels were significantly elevated after conditioning $(p<0.001)$ and there was no main effect of phenotype: bHRs showed similarly high corticosterone levels with all other phenotypes $(\mathrm{F}(2,41)=0.82, p=0.45$, Figure 2e). There was no significant interaction between collection time and phenotype $(p=0.45)$ : all phenotypes demonstrated the same rise in corticosterone levels between baseline and conditioning time points. Together, these data suggest that all groups exhibit similarly high stress levels postconditioning; therefore, we explored extinction learning in these different lines of animals.

bLRs exhibit reduced fear extinction learning, retention, and increased contextual fear. We first confirmed that our paradigm generated extinction learning by examining the main effect of trial $(\mathrm{F}(4.47,402.62)=11.53, \quad p<0.001$; Figure 3a). Animals displayed higher freezing during the first two tone-bins of the session (post hoc tests comparing tone-bins 5 and 10 with all subsequent bins $p<0.01$ ), indicating that extinction learning occurred (Figure $3 \mathrm{a}$ ). The data supported our hypothesis, that the phenotypes would respond differentially to extinction learning, with a main effect of phenotype $(\mathrm{F}(2,90)=23.67, p<0.001$; Figure $3 \mathrm{~b})$ : bHRs displayed significantly lower freezing (post hoc tests $p<0.001)$ while bLRs showed significantly higher freezing (post hoc tests $p<0.005$ ) than all other groups. We also confirmed the main effect of extinction group $(\mathrm{F}(1,90)$ $=44.42, p<0.001$; Supplementary Figure 2B): rats undergoing extinction had increased freezing relative to NoExtinction rats. As expected, there was a trial by extinction group interaction: the pattern of freezing for Extinction and No-Extinction rats differed throughout the session, because Extinction rats showed a reduction in fear over time $(\mathrm{F}(4.45,391.83)=19.50, p<0.001$; Supplementary Figure 2A and $\mathrm{B})$. No other effects reached statistical significance. Further experiments using our selectively bred animals indicate that this phenotype effect is consistent across generations, demonstrating that these behaviors are inherited and predictable (Supplementary Figure 3).

To complement our analysis of fear behavior during conditioning, we explored escape behavior and corticosterone levels during extinction learning. During extinction, 


\section{Conditioning}
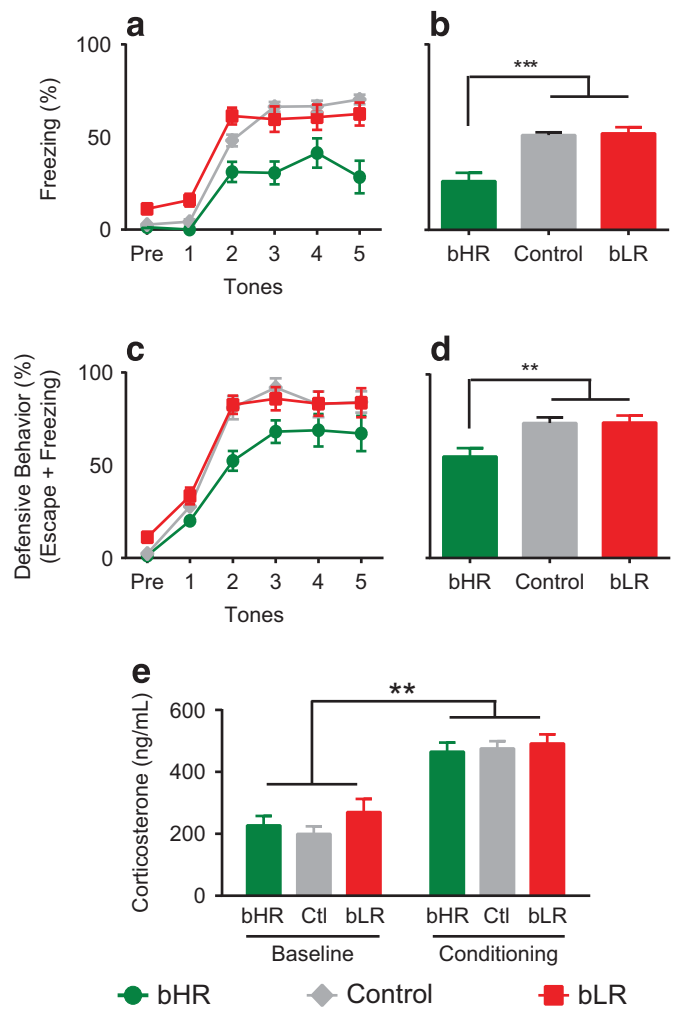

Figure 2 Rats selectively bred for locomotion in a novel environment display individual differences in fear conditioning behavior. During fear conditioning, bHR rats display lower levels of freezing (two-way repeatedmeasures ANOVA followed by Bonferroni-corrected post hoc test for phenotype $p<0.00$ I compared with both bLR and control rats; a). This can be seen in the significant main effect of phenotype displayed as average freezing across the five tones (two-way repeated-measures ANOVA: main effect of phenotype $F(2,90)=23.52, p<0.00 \mathrm{I} ; \mathrm{b}$ ). When escape behaviors are included with freezing as a measure of defensive behavior, bHR fear levels are more similar to those of bLR and controls (two-way repeatedmeasures ANOVA followed by Bonferroni-corrected post hoc test for phenotype $p=0.04$ for bLR and $p=0.12$ for controls; $c$ ), though the main effect of phenotype is still significant (two-way repeated-measures ANOVA: main effect of phenotype $F(2,42)=3.82, p=0.03$; d). Although freezing behavior differs by phenotype, there are no phenotype differences in corticosterone levels before or after conditioning (one-way repeated measures ANOVA: main effect of phenotype $F(2,4 I)=0.82, p=0.45$; e). This indicates that stress levels may be similar across phenotypes, even though freezing is not the preferred defensive behavior of bHR animals. ( $N=16$ bHR, bLR; $N=64$ controls) $* * * * p<0.00$ I, ** $p<0.01$.

bHRs again displayed more escape behavior. However, this effect was primarily observed in No-Extinction animals in a phenotype by extinction group interaction, demonstrating that escape behavior is not an important variable in the low freezing behavior of bHRs during extinction learning $(\mathrm{F}(2,42)=5.00, p=0.011$; Supplementary Figure $2 \mathrm{C})$. Analysis of corticosterone levels showed a significant increase over baseline after extinction learning $(\mathrm{F}(1,20)=45.49, p<0.001)$ but no interaction with phenotype $(p=0.258$; Supplementary Figure 2D).

We confirmed bLRs' reduced extinction learning by testing their retention of extinction learning $24 \mathrm{~h}$ later. bLRs showed high freezing compared with other phenotypes during retention testing (Figure 3c; Supplementary
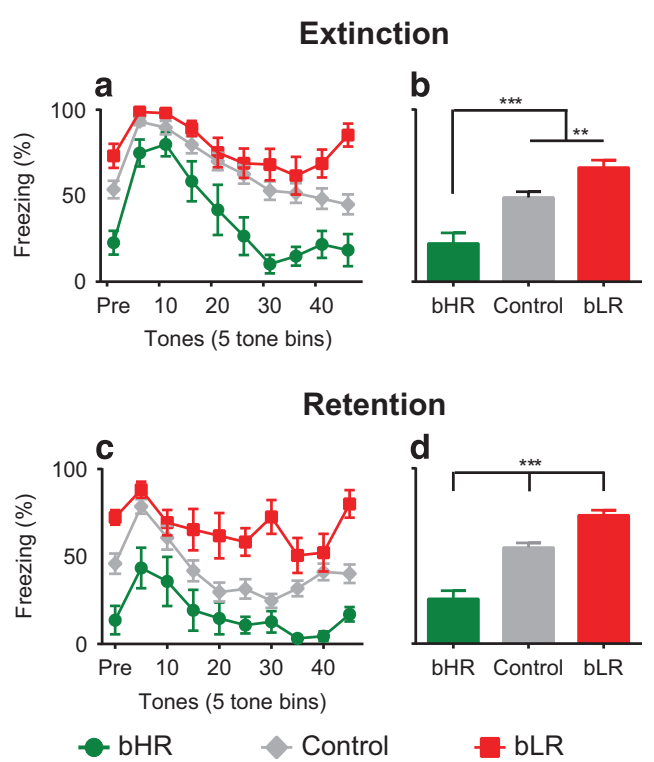

Figure 3 Selectively bred rats display distinct differences in extinction and retention behavior. During extinction learning, bHRs appear to show facilitated within-session learning compared with controls while bLRs show a slight reduction in extinction learning (a). This is confirmed by a significant main effect of phenotype where bHR freezing levels are significantly different from both control and bLR freezing levels, which also differ from each other, with bLRs having the highest freezing (two-way repeated measures ANOVA: main effect of phenotype $F(2,90)=23.67, p<0.00$ I, post hoc tests bHRs $p<0.00$ I, bLRs $p<0.005$ for all; b). Extinction retention reveals stark differences in freezing behavior, with bLRs showing strongly reduced extinction retention compared with controls and bHRs showing facilitated retention compared with controls (two-way repeated-measures ANOVA followed by Bonferroni-corrected post hoc tests; c). This is confirmed by a main effect of phenotype, where all phenotypes are significantly different from each other with bLRs showing the least retention (two-way repeatedmeasures ANOVA: main effect of phenotype $F(2,90)=34.16, p<0.001$, Bonferroni-corrected post hoc tests bHR $<$ controls $p<0.00 \mathrm{I}$ and controls $<$ bLR $p<0.00$ I; d). The reduced extinction learning and retention evidenced by bLR rats indicates a more PTSD-like behavioral phenotype, while bHR rats seem to be particularly resilient. ( $N=8$ bHRs, bLRs; $N=32$ Controls) ***** $p<0.001$, *** $p<0.01$.

Figure 4A). We confirmed further extinction learning during our retention test using the main effect of trial (F $(3.51,308.86)=28.90, \quad p<0.001$; Figure $3 \mathrm{c})$ : all animals demonstrated significantly higher freezing during the first two tone-bins of the session, indicating that their fear once again decreased over time (post hoc tests comparing tonebins 5 and 10 with all subsequent bins $p<0.015$ ). The main effect of phenotype $(\mathrm{F}(2,90)=34.16, p<0.001$; Figure $3 \mathrm{~d})$ demonstrated that bHRs showed lower freezing than controls (post hoc $p<0.001$ ), which were significantly lower than bLRs (post hoc $p<0.001$ ). This confirmed that bLRs demonstrate reduced extinction learning. In further confirmation of the success of extinction learning, No-Extinction animals that had not received extinction the day before showed higher freezing than Extinction animals in a main effect of extinction group during retention, demonstrating that extinction learning was retained overall $(\mathrm{F}(1,88)=29.01$, $p<0.001$; Supplementary Figure 4B). No other effects reached statistical significance.

When rats were placed back in the conditioning context after extinction learning and retention testing, there was a 


\section{Locomotion}

a

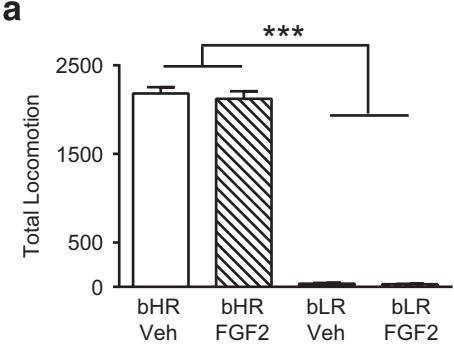

\section{b}

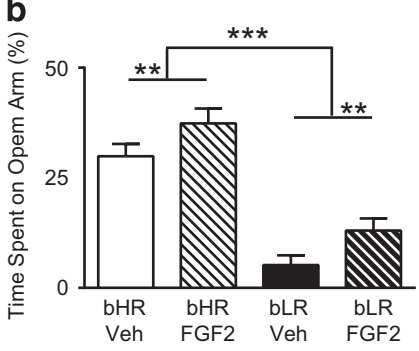

Elevated Plus Maze

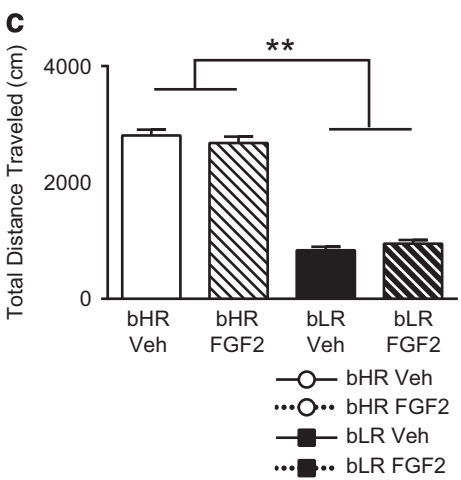

Conditioning

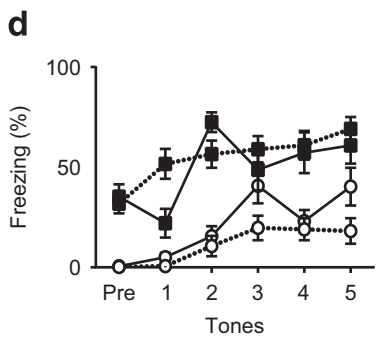

e

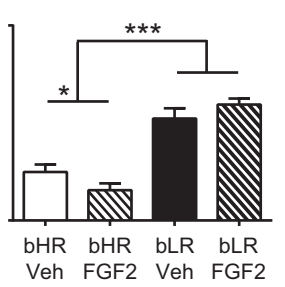

f

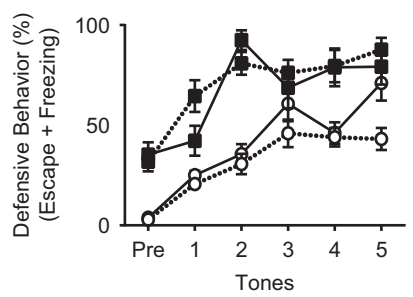

g

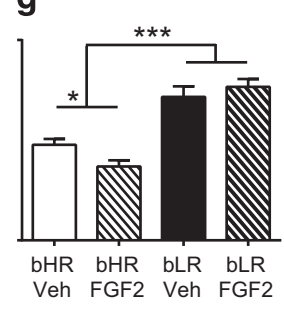

Figure 4 Early-life administration of FGF2 does not affect locomotor behavior, decreases spontaneous anxiety, and has a mild effect on fear learning in bHR animals. Although large differences in phenotypic locomotor response to novelty remain, with bHRs showing greater locomotion than bLRs, there is no effect of early-life FGF2 administration on locomotion for either phenotype (two-way ANOVA: main effect of phenotype $F(I, 57)=\mid 22.26$, $p<0.001$, Bonferronicorrected post hoc test $p<0.00 \mathrm{I}$, main effect of treatment $p=0.693$; a). Administration of FGF2 on the day after birth reduced anxiety-like behavior in adulthood, as shown by an increase in the percentage of time spent in the open arms of the elevated plus maze for both bHR and bLR animals (two-way ANOVA: main effect of treatment $F(I, 54)=6.88, p=0.01 \mathrm{I}$, FGF2 $<$ Veh; b). Although the phenotype difference remains clear between the two lines of animals, early-life FGF2 did not change the distance traveled from vehicle levels for either bHRs or bLRs, indicating that the anxiolytic effects of FGF2 are specific and not due to a general increase in locomotor behavior (two-way ANOVA: main effect of phenotype $F(1,57)=395.03, p<0.001$, main effect of treatment $p=0.874 ; c)$. Administration of FGF2 the day after birth does not change fear learning in bLR animals; however, it does reduce freezing in bHR animals by a small amount (three-way repeated-measures ANOVA: treatment by phenotype interaction $F(I, 5 \mathrm{I})=7.59$, $p=0.008$, Bonferroni-corrected post hoc test $p=0.034 ; d)$. Similar to our previous study, bHRs show reduced freezing behavior, creating a main effect of phenotype (three-way repeated-measures ANOVA: main effect of phenotype $F(I, 5 I)=89.60, p<0.00 I$ bHR $<b L R ;$ e). There is also a significant treatment by phenotype interaction, with bHRs showing a further reduction in freezing behavior after early-life FGF2 administration (three-way repeated-measures ANOVA: treatment by phenotype interaction $F(I, 5 I)=7.59, p=0.008$, Bonferroni-corrected post hoc test $p=0.034$; e). Defensive behavior continues to demonstrate a difference between bHR and bLR animals during conditioning (three-way repeated-measures ANOVA: main effect of phenotype $F(I, 5 I)=69.945, p<0.00 \mathrm{I}, \mathrm{bHR}<\mathrm{bLR} ; \mathrm{f}$ ), but the FGF2 by phenotype interaction is less significant (three-way repeated-measures ANOVA: treatment by phenotype interaction $\mathrm{F}(\mathrm{I}, 5 \mathrm{I})=4.3 \mathrm{I} 4, p=0.04 ; \mathrm{g}$ ). $(N=16$ bHR FGF2; $N=14$ bHR Veh, bLR FGF2; $N=13$ bLR Veh) $* * * *<<0.001$, $* * * 0.01$, $* p<0.05$

significant main effect of phenotype: bLRs displayed higher freezing than either bHR or control animals suggesting that their contextual fear, similar to their cued fear, does not diminish over time $(\mathrm{F}(2,90)=80.91, p<0.001$; post hoc tests all $p<0.001$; Supplementary Figure $5 \mathrm{~A}$ and $\mathrm{B}$ ).

\section{Experiment 2}

Early-life FGF2 administration reduces spontaneous anxiety-like behavior on EPM. In a second experiment, we confirmed that early-life administration of FGF2 had no effect on locomotor behavior or phenotype differences $(p=0.693$, Figure 4a). As previously observed, there remained a highly significant main effect of phenotype on locomotor behavior $(\mathrm{F}(1,57)=1227.26, p<0.001)$ : bHRs had significantly higher locomotor response than bLRs (post hoc $p<0.001)$. However, early-life FGF2 administration produced a significant main effect of treatment on the EPM (F $(1,54)=6.88, p=0.011$; Figure $4 \mathrm{~b})$ : relative to vehicle, rats that received early-life FGF2 spent more time in the open arms of the EPM. As expected, the significant main effect of phenotype remained: bHRs spent significantly more time on the open arms than bLRs $(\mathrm{F}(1,54)=61.30, p<0.001$; Figure $4 \mathrm{~b})$. There was no effect of FGF2 on the total distance traveled, indicating that the effects of FGF2 are specific to anxiety-like behavior (main effect of treatment $p=0.874$; Figure 4c).

Early-life FGF2 has a subtle effect on fear learning. During conditioning, we again confirmed that our conditioning paradigm successfully generated learning using the main effect of trial $(\mathrm{F}(4,204)=11.31, p<0.001$; Figure $4 \mathrm{~d})$. Animals displayed significantly higher freezing levels to tones 2-5 relative to tone 1 (post hoc $p<0.001$ ). Similar to Experiment 1, the significant main effect of phenotype $(\mathrm{F}(1,51)=89.60, p<0.001)$ showed that bHRs have lower freezing than bLRs (Figure 4e) during conditioning. Also, as in Experiment 1, there was a significant interaction of trial by phenotype $(\mathrm{F}(4,204)=2.78, p=0.028)$ : bHRs demonstrated a more gradual increase in freezing than bLRs during conditioning. A significant interaction between treatment and phenotype $(\mathrm{F}(1,51)=7.59, p=0.008)$ demonstrated that 
FGF2 affects bHR but not bLR freezing during conditioning; however, there was no main effect of treatment $(p=0.853$; Figure 4e). When freezing and escape were combined to create a general measure of defensive behavior (Figure 4f), the treatment by phenotype interaction remained significant $(\mathrm{F}(1,51)=4.314, p=0.04$; Figure $4 \mathrm{~g})$, indicating that early-life FGF2 administration mildly reduces bHR freezing. As in Experiment 1, there was a significant main effect of phenotype on escape behavior $\mathrm{F}(1,53)=9.168, p=0.004)$ : bHRs showed greater escape behavior than bLRs (Supplementary Figure 6A). There were no other significant effects, indicating that escape behavior did not account for the differential freezing behavior observed in early-life FGF2injected bHR animals. A separate cohort of bIR animals showed no main effect of FGF2 treatment on fear conditioning, indicating that the effect of FGF2 is specific to bHRs $\quad(\mathrm{F}(1,24)=3.132, \quad p=0.09$; $\quad$ Supplementary Figure 6B).

Early-life FGF2 administration facilitates fear extinction for resilient animals in adulthood. We first confirmed that our extinction paradigm generated learning because the main effect of trial $(\mathrm{F}(5.95,291.77)=2.84, \quad p=0.011$; Figure 5a) indicated that freezing decreased across the session. Similar to Experiment 1, there was a significant trial by phenotype interaction $(\mathrm{F}(5.95,291.77)=10.158, p<0.001$; Figure 5a): bLRs maintained a pattern of freezing throughout the session, while bHRs demonstrated extinction learning (post hoc tests between all tone-bins by phenotype $p<0.01$ ). As expected from Experiment 1, bHRs again displayed lower freezing than bLRs overall in a main effect of phenotype $(\mathrm{F}(1,49)=247.34, p<0.001$; Figure $5 \mathrm{~b})$. There was a trend toward a main effect of treatment $(\mathrm{F}(1,49)=3.22, p=0.079)$ : vehicle animals showed higher freezing than FGF2 animals, indicating that early-life administration of FGF2 facilitated extinction learning in adulthood (Figure 5b). There was a trend toward a phenotype by treatment interaction on area under the curve $(\mathrm{F}(1,49)=3.24, p=0.078)$, indicating that early-life administration of FGF2 facilitated extinction learning, particularly in bHRs, over the course of the extinction session. As expected, we further confirmed that Extinction animals displayed significantly higher freezing compared with No-Extinction animals in a main effect of extinction group $(\mathrm{F}(1,49)=58.92, p<0.001$; Supplementary Figure $7 \mathrm{~B}$ ). The pattern of freezing across the session indicated that extinction learning occurred in the Extinction animals in a significant interaction between trial and extinction group $(\mathrm{F}(5.95,291.77)=23.007, \quad p<0.001$; Supplementary Figure 7A and B). A separate cohort of bIR animals had no main effect of FGF2 treatment on extinction learning $\quad(\mathrm{F}(1,24)=3.102, \quad p=0.091 ; \quad$ Supplementary Figure 7C), indicating that the impact of FGF2 seems again to be specific to bHRs under these conditions.

During extinction retention testing, the main effect of trial indicated learning occurred $(\mathrm{F}(4.76,233.34)=28.00$, $p<0.001$; Figure $5 \mathrm{c}$ ): animals froze significantly more during the first two tone-bins of the session (post hoc tests comparing tone-bins 5 and 10 , all subsequent tone-bins $p<0.001)$. Similar to Experiment 1, bHRs showed a steeper decline in freezing across the session than bLRs as demonstrated by a significant interaction between trial and
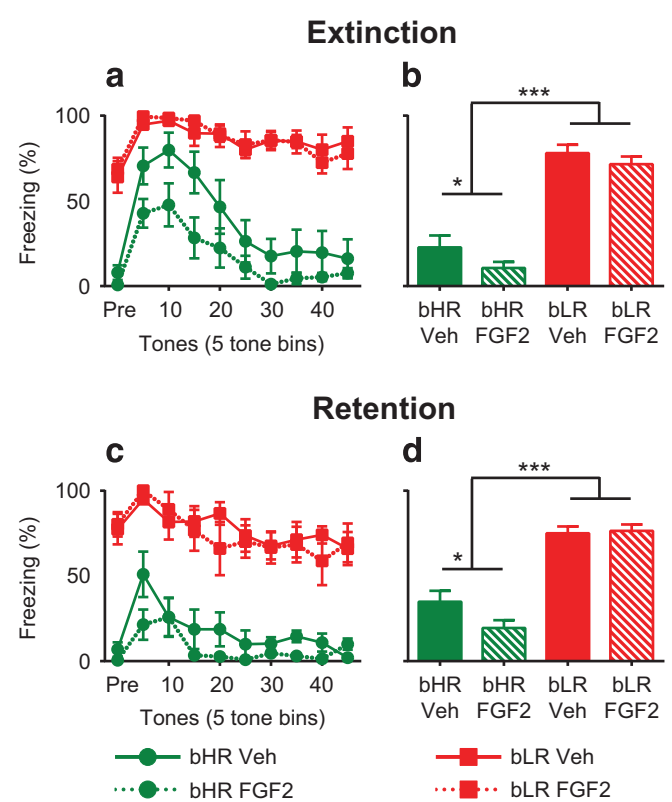

Figure 5 Early-life administration of FGF2 facilitates extinction learning and retention only in more resilient bHR animals. Administration of FGF2 the day after birth does not change fear extinction in bLR animals; however, it does reduce freezing in bHR animals by a small amount (three-way repeated-measures ANOVA: trend toward main effect of treatment $F$ $(I, 49)=3.22, p=0.079 ; a)$. The main effect of phenotype on freezing and the trend toward a phenotype by treatment interaction (post hoc test $p<0.05$ for bHRs) demonstrate facilitated extinction in bHRs and particularly for bHRs given early-life FGF2 (three-way repeated-measures ANOVA: main effect of phenotype $F(I, 49)=247.34, p<0.001$, bHR $<$ bLR; b). During extinction retention, bHRs given FGF2 early in life show facilitated extinction retention, while there is no effect on bLRs (three-way repeatedmeasures ANOVA: phenotype by treatment interaction $F(I, 49)=4.69$, $p=0.035$, Bonferroni-corrected post hoc test bHR FGF2 <bHR Veh $p<0.05 ; c)$. This can be seen both in the main effect of phenotype and also the significant phenotype by treatment interaction (post hoc test $p<0.05$ for bHRs) where bHRs demonstrated facilitated extinction retention, particularly when given early-life FGF2 (three-way repeatedmeasures ANOVA: main effect of phenotype $F(I, 49)=134.78, p<0.00 I$, $\mathrm{bHR}<\mathrm{bLR}$, phenotype by treatment interaction $\mathrm{F}(\mathrm{I}, 49)=4.69, p=0.035$, Bonferroni-corrected post hoc test bHR FGF2 <bHR Veh $p<0.05$; d). These data demonstrate that individual differences in response to novelty can affect the efficacy of treatment with FGF2 to facilitate extinction learning and retention. ( $N=8$ bHR FGF2, bLR Veh; $N=7$ for bHR Veh, bLR FGF2) ***** $p<0.00$ I, *** $p<0.0$ I, * post hoc $p<0.05$.

phenotype $(\mathrm{F}(4.76,233.34)=3.27, p=0.008$; Figure $5 \mathrm{c})$. There was a main effect of phenotype similar to Experiment 1: bHRs had significantly lower freezing than bLRs overall $(\mathrm{F}(1,49)=134.78, p<0.001$; Figure $5 \mathrm{~d})$. Importantly, earlylife FGF2 administration reduced freezing compared with vehicle animals for bHRs, but not for bLRs, indicating that FGF2 facilitated extinction retention specifically in bHRs in a phenotype by treatment interaction $(\mathrm{F}(1,49)=4.69$, $p=0.035$; Figure $5 \mathrm{~d}$ ). We confirmed a significant main effect of extinction group: Extinction animals displayed lower freezing relative to No-Extinction animals demonstrating retention of their extinction learning $(\mathrm{F}(1,49)=10.46$, $p=0.002$; Supplementary Figure $8 \mathrm{~B})$. No-Extinction animals had a higher peak and steeper curve than animals that had already undergone extinction in a trial by extinction group interaction, demonstrating that extinction learning differs 
from the pattern of freezing exhibited by animals that have already learned extinction $(\mathrm{F}(4.76,233.34)=2.79, p=0.020$; Supplementary Figure 8A and B). As expected, based on the results of Experiment 1, bHR Extinction animals retained their extinction learning from the day before while bLRs demonstrated no difference between Extinction and No-Extinction in a phenotype by extinction group interaction $(\mathrm{F}(1,49)=12.90, p=0.001$; Supplementary Figure $8 \mathrm{C})$. The significant trial by phenotype by extinction group interaction $(\mathrm{F}(4.76,233.34)=3.31, p=0.008$; Supplementary Figure 8C) indicated the freezing pattern of the bLR Extinction and No-Extinction groups differed across the session from those of the bHR Extinction and No-Extinction groups further demonstrating that bHRs retain their extinction learning while bLRs do not. A separate cohort of bIR animals showed no main effect of FGF2 treatment during retention $(\mathrm{F}(1,24)=2.681, p=0.115$; Supplementary Figure $8 \mathrm{D}$ ), demonstrating that the effect of FGF2 is again specific to bHR animals.

When placed back in the conditioning context after extinction and extinction retention, there was a significant main effect of phenotype similar to Experiment 1: bLRs displayed higher freezing than bHRs $(\mathrm{F}(1,48)=718.68$, $p<0.001$; Supplementary Figure $9 \mathrm{~A}$ and $\mathrm{B})$. There was no main effect of treatment (bHR/bLR, $\mathrm{F}(1,48)=0.65, p=0.42)$ for any phenotype (bIRs, $\mathrm{F}(1,24)=0.04, p=0.84$ ). This indicates that there is no effect of FGF2 on contextual fear for any phenotype, although FGF2 does impact bHR behavior during extinction and extinction retention.

\section{DISCUSSION}

Selectively bred differences in response to novelty may predict individual differences in PTSD-like behaviors. During fear conditioning, bHRs displayed more escape behavior, while bLRs showed higher levels of freezing behavior. This differential response profile has some parallels with active and passive defensive responses in humans (Blanchard et al, 2001; Harrison et al, 2015). Although fear conditioning in selectively bred animals was similar to outbred animals, bLRs show reduced extinction learning and retention, a feature also characteristic of human PTSD patients (Blechert et al, 2007; Milad et al, 2009; Norrholm et al, 2011; Orr et al, 2000). Furthermore, we demonstrated that a single administration of FGF2 the day after birth facilitated extinction retention for bHRs but not for bLRs, though it reduced spontaneous anxiety. Collectively, these results inform how variation in temperament may influence the tendency to develop PTSD and indicate that FGF2 treatment may impact individuals differentially.

\section{bHRs and bLRs Show Behavioral Differences During Fear Conditioning and Extinction}

Our studies provide evidence that bLR rats present a unique model of vulnerability to PTSD. In humans, a history of depression and a negative bias in cognition have been linked to increased risk for developing PTSD (Bomyea et al, 2012; Orr et al, 2012). Human patient populations also display reduced extinction learning (Blechert et al, 2007; Lommen et al, 2013; Norrholm et al, 2011; Orr et al, 2000). Previous studies showed that bLRs display high levels of spontaneous anxiety- and depression-like behavior (Perez et al, 2009; Stead et al, 2006; Turner et al, 2011), and here we observe reduced extinction learning and retention and increased contextual fear compared with outbred animals and bHRs. This trait may generalize: outbred LRs identified by their locomotion recently showed reduced extinction learning compared with outbred HRs (Duclot et al, 2016). Therefore, reduced extinction learning and retention demonstrated by bLR rats likely indicates an increased propensity to develop PTSD-like behavior. Our model generates a consistently vulnerable phenotype useful for experimental testing; however, it may not model individuals that display characteristics of vulnerability but do not develop PTSD.

In contrast, bHR animals may provide a model of resilience to developing PTSD. During conditioning, bHRs exhibited more active escape responses during the conditioning session. Active coping in humans correlated with reduced PTSD symptoms in trauma-exposed veterans (Contractor et al, 2015). Similarly, we observed facilitated extinction learning in bHR animals that was not due to increased escape behavior, indicating that active coping during fear conditioning may contribute to lower levels of fear retention and PTSD-like behavior. bHR animals have high endogenous expression of FGF2 in the hippocampus (Perez et al, 2009), and consistent with this, outbred animals with higher levels of endogenous FGF2 in the hippocampus show lower levels of fear in contextual and cued fear conditioning (Graham and Richardson, 2016). bHR NoExtinction animals also show very low freezing, and bHRs overall demonstrate little contextual fear. Further studies should explore contextual discrimination, especially as bLRs appear to overgeneralize their contextual fear. Overall, bHRs demonstrate enhanced extinction learning and retention over controls, indicating that they are particularly resilient to developing PTSD-like behaviors.

As our animals are selectively bred based on locomotion, movement in the novel fear conditioning chambers could be a potential confound in these studies. However, our observations suggest that this cannot fully explain our results. Although bLRs move less in a novel environment than outbred animals, their freezing levels prior to fear conditioning were $<20 \%$ and freezing decreased during the extinction and retention sessions, indicating that their immobility is likely due to fear and not purely due to their phenotypic lack of locomotion. Corticosterone levels support this interpretation, demonstrating increased activation of the HPA axis after both fear conditioning and extinction. Likewise, facilitated fear extinction in bHRs was not solely due to increased locomotion, as freezing levels at the start of the extinction session were very close to those of outbred animals. Moreover, escape behavior did not increase across the extinction session, and escape behaviors were not elevated in Extinction bHR animals. Fecal bolus data showed no phenotype differences during any behavioral session, indicating that this measure does not correlate with freezing behavior for these animals. Although we believe that the data described here provide strong evidence for phenotypic differences in fear behavior, future studies should further assess differences in response to fear stimuli in selectively bred rodents using other measures. 


\section{Early-Life FGF2 Facilitates Fear Extinction Only in Resilient bHR Animals}

As we develop better models of individual differences in PTSD-like behavior, we can investigate the role of those intrinsic differences in responsiveness to potential prevention and/or treatment options. Graham and Richardson $(2009,2010)$ previously reported that acute administration of FGF2 enhanced extinction learning and retention and reduced the return of fear after extinction. This indicates that FGF2 might be a promising pharmacological tool to enhance extinction learning (Graham and Richardson, 2015). We have previously shown that FGF2 reduces anxiety-like behavior in adults (Perez et al, 2009) and has an organizational role in development to alter emotional responsiveness and impact epigenetic factors (Chaudhury et al, 2014). Therefore, we asked whether FGF2 could also mediate fear extinction during adulthood when administered at a developmental time point.

We assessed whether a single administration of FGF2 during early life enhanced extinction learning and retention in adulthood and whether this differed between bHR, bIR, and bLR rats. Replicating previous findings (Turner et al, 2011), we observed that early-life FGF2 reduced spontaneous anxiety in bLRs, though bHRs also showed the effect. Contrary to our hypothesis, only bHRs demonstrated any effect of FGF2 on their fear extinction behavior. bHRs administered FGF2 showed a mild enhancement of extinction learning and a significant enhancement of extinction retention. In contrast, early-life FGF2 treatment had no effect on bLR or bIR behavior during extinction or retention, and no phenotypes demonstrated effects of early-life FGF2 on contextual fear behavior. This indicates that, under the current paradigm, early-life FGF2 administration reduced cued fear responses during extinction learning and retention selectively in resilient individuals. Although FGF2 is able to modulate mild anxiety responses in vulnerable animals (eg, EPM), it is insufficient to dampen strong resistance to extinction learning in our hands. Interestingly, Graham and Richardson (2009) previously showed that administration of FGF2 did not enhance extinction retention when extinction learning did not occur. This may partially explain why bLRs showed no effect of early-life FGF2 administration: because they showed little evidence of learning extinction, FGF2 could not enhance their retention. Alternatively, the effect of FGF2 on extinction in bLRs may have been hidden by a ceiling effect, though we believe this to be unlikely given that bLR freezing decreased during the retention session without displaying an effect of FGF2 treatment. Although the underlying cause remains unclear, these data demonstrate that FGF2 affects individuals differentially based on the type of testing: it reduces anxiety but not conditioned fear in vulnerable animals, though it facilitates extinction in resilient animals.

Although early-life administration of FGF2 had no effect on extinction in the vulnerable bLR animals, there remain other possibilities for testing pharmacological enhancers of extinction, such as d-cycloserine, which can facilitate extinction in rats (Fitzgerald et al, 2014) and is effective at reducing PTSD behaviors in humans (Difede et al, 2014). Additional studies may also explore whether fear conditioning and extinction differentially modulate FGF2 expression in our rodent model. Future studies should also demonstrate whether these individual differences in fear learning, extinction learning, and retention also differentiate responses to the return of fear after extinction. For example, it is unclear whether bHRs would demonstrate renewal (the return of fear in a new context) or reinstatement (the return of fear after experiencing a fear reminder) compared with outbred animals, especially given their low levels of contextual fear. Additional studies could also explore multiple conditioning sessions to model repeated stress in PTSD. Although the genetic underpinnings of the differences between bHRs and bLRs are currently under study, it is likely that a host of genes contribute to the differences in emotional vulnerability and resilience between the lines. Thus further study of the bHR animals may lead to insights into the molecular and neural underpinnings of their resilience that can be used to manipulate the vulnerability shown by bLRs.

\section{Summary}

We have developed an animal model that demonstrates differences in PTSD-like behavior that are markedly similar to the differences observed in humans. We also demonstrated that a single administration of FGF2 early in life facilitates extinction in resilient animals, although it fails to do so in vulnerable animals and controls. This suggests that it may be important to tailor the treatment or prevention strategy to different individuals. Future work will examine how the model resembles human disease and whether other interventions may be effective at reducing the behavioral vulnerability that bLRs exhibit.

\section{FUNDING AND DISCLOSURE}

Funding for this work comes from the Office of Naval Research grants N00014-09-1-0598 and N00014-12-1-0366, the National Institute of Mental Health R01MH104261, the Pritzker Neuropsychiatric Disorders Research Consortium, and the Hope for Depression Research Foundation (HDRF). SM was supported by NIH grant R01MH065961 and a McKnight Memory and Cognitive Disorders Award. KEP was supported by a Rackham Predoctoral Fellowship as well as training grants NIH T-32-NS076401, and NIMH 5-T-32MH014279. ELA was supported by the National Science Foundation Graduate Research Fellowship Program as well as training grants NIH T-32-DA007281-17 and T-32EY017878-10. The authors declare no conflict of interest.

\section{ACKNOWLEDGMENTS}

We thank Pamela Maras for assistance with early-life FGF2 injections. We also thank Angela Koelsch, Jim Stewart, and Fei Li for their assistance with the colony of selectively bred animals and technical assistance; Amy Tang and Jennifer Fitzpatrick for their technical assistance; and Jonathan Morrow, Tom Dixon, Jim Burton, Loren Rector, and Doug Smith for their assistance with the fear conditioning equipment and recording setup. 


\section{REFERENCES}

Aurbach EL, Inui EG, Turner CA, Hagenauer MH, Prater KE, Li JZ et al (2015). Fibroblast growth factor 9 is a novel modulator of negative affect. Proc Natl Acad Sci USA 112: 11953-11958.

Blanchard DC, Hynd AL, Minke KA, Minemoto T, Blanchard RJ (2001). Human defensive behaviors to threat scenarios show parallels to fear- and anxiety-related defense patterns of nonhuman mammals. Neurosci Biobehav Rev 25: 761-770.

Blechert J, Michael T, Vriends N, Margraf J, Wilhelm FH (2007). Fear conditioning in posttraumatic stress disorder: evidence for delayed extinction of autonomic, experiential, and behavioural responses. Behav Res Ther 45: 2019-2033.

Bomyea J, Risbrough V, Lang AJ (2012). A consideration of select pre-trauma factors as key vulnerabilities in PTSD. Clin Psychol Rev 32: 630-641.

Chaudhury S, Aurbach EL, Sharma V, Blandino P Jr., Turner CA, Watson SJ et al (2014). FGF2 is a target and a trigger of epigenetic mechanisms associated with differences in emotionality: partnership with H3K9me3. Proc Natl Acad Sci USA 111: 11834-11839.

Contractor AA, Armour C, Shea MT, Mota N, Pietrzak RH (2015). Latent profiles of DSM-5 PTSD symptoms and the 'Big Five' personality traits. J Anxiety Disord 37: 10-20.

Difede J, Cukor J, Wyka K, Olden M, Hoffman H, Lee FS et al (2014). D-cycloserine augmentation of exposure therapy for post-traumatic stress disorder: a pilot randomized clinical trial. Neuropsychopharmacology 39: 1052-1058.

Duclot F, Perez-Taboada I, Wright KN, Kabbaj M (2016). Prediction of individual differences in fear response by novelty seeking, and disruption of contextual fear memory reconsolidation by ketamine. Neuropharmacology 109: 293-305.

Fitzgerald PJ, Seemann JR, Maren S (2014). Can fear extinction be enhanced? A review of pharmacological and behavioral findings. Brain Res Bull 105: 46-60.

Flagel SB, Waselus M, Clinton SM, Watson SJ, Akil H (2014). Antecedents and consequences of drug abuse in rats selectively bred for high and low response to novelty. Neuropharmacology 76 (Pt B): 425-436.

Graham BM, Richardson R (2009). Acute systemic fibroblast growth factor-2 enhances long-term extinction of fear and reduces reinstatement in rats. Neuropsychopharmacology 34: 1875-1882.

Graham BM, Richardson R (2010). Fibroblast growth factor-2 enhances extinction and reduces renewal of conditioned fear. Neuropsychopharmacology 35: 1348-1355.

Graham BM, Richardson R (2011). Memory of fearful events: the role of fibroblast growth factor-2 in fear acquisition and extinction. Neuroscience 189: 156-169.

Graham BM, Richardson R (2015). Fibroblast growth factor 2 as a new approach to fighting fear. JAMA Psychiatry 72: 959-960.

Graham BM, Richardson R (2016). Individual differences in the expression of conditioned fear are associated with endogenous fibroblast growth factor 2. Learn Mem 23: 42-45.

Harrison LA, Ahn C, Adolphs R (2015). Exploring the structure of human defensive responses from judgments of threat scenarios. PLOS ONE 10: e0133682.

Holly EN, Miczek KA (2015). Capturing individual differences: challenges in animal models of posttraumatic stress disorder and drug abuse. Biol Psychiatry 78: 816-818.

Jaccard J, Becker MA (2009). Statistics for the Behavioral Sciences, 5th edn. Wadsworth/Thomson Learning: Belmont, CA, USA, 736 pp.

Kerman IA, Clinton SM, Bedrosian TA, Abraham AD, Rosenthal DT, Akil $\mathrm{H}$ et al (2011). High novelty-seeking predicts aggression and gene expression differences within defined serotonergic cell groups. Brain Res 1419: 34-45.

Kessler RC, Sonnega A, Bromet E, Hughes M, Nelson CB (1995). Posttraumatic stress disorder in the National Comorbidity Survey. Arch Gen Psychiatry 52: 1048-1060.

Khan AA, Jacobson KC, Gardner CO, Prescott CA, Kendler KS (2005). Personality and comorbidity of common psychiatric disorders. Br J Psychiatry 186: 190-196.

Lommen MJ, Engelhard IM, Sijbrandij M, van den Hout MA, Hermans D (2013). Pre-trauma individual differences in extinction learning predict posttraumatic stress. Behav Res Ther 51: 63-67.

Maren S, Phan KL, Liberzon I (2013). The contextual brain: implications for fear conditioning, extinction and psychopathology. Nat Rev Neurosci 14: 417-428.

Milad MR, Pitman RK, Ellis CB, Gold AL, Shin LM, Lasko NB et al (2009). Neurobiological basis of failure to recall extinction memory in posttraumatic stress disorder. Biol Psychiatry 66: $1075-1082$.

Milad MR, Rosenbaum BL, Simon NM (2014). Neuroscience of fear extinction: implications for assessment and treatment of fearbased and anxiety related disorders. Behav Res Ther 62: 17-23.

Norrholm SD, Jovanovic T, Olin IW, Sands LA, Karapanou I, Bradley B et al (2011). Fear extinction in traumatized civilians with posttraumatic stress disorder: relation to symptom severity. Biol Psychiatry 69: 556-563.

Orr SP, Lasko NB, Macklin ML, Pineles SL, Chang Y, Pitman RK (2012). Predicting post-trauma stress symptoms from pre-trauma psychophysiologic reactivity, personality traits and measures of psychopathology. Biol Mood Anxiety Disord 2: 8.

Orr SP, Metzger LJ, Lasko NB, Macklin ML, Peri T, Pitman RK (2000). De novo conditioning in trauma-exposed individuals with and without posttraumatic stress disorder. J Abnorm Psychol 109: 290-298.

Perez JA, Clinton SM, Turner CA, Watson SJ, Akil H (2009). A new role for FGF2 as an endogenous inhibitor of anxiety. J Neurosci 29: 6379-6387.

Stead JD, Clinton S, Neal C, Schneider J, Jama A, Miller S et al (2006). Selective breeding for divergence in novelty-seeking traits: heritability and enrichment in spontaneous anxiety-related behaviors. Behav Genet 36: 697-712.

Turner CA, Clinton SM, Thompson RC, Watson SJ Jr., Akil H (2011). Fibroblast growth factor-2 (FGF2) augmentation early in life alters hippocampal development and rescues the anxiety phenotype in vulnerable animals. Proc Natl Acad Sci USA 108: 8021-8025.

Turner CA, Gula EL, Taylor LP, Watson SJ, Akil H (2008). Antidepressant-like effects of intracerebroventricular FGF2 in rats. Brain Res 1224: 63-68.

Supplementary Information accompanies the paper on the Neuropsychopharmacology website (http://www.nature.com/npp) 